\title{
Existence and multiplicity of positive solutions of a one-dimensional mean curvature equation in Minkowski space
}

Minghe Pei, Libo Wang* and Xuezhe Lv

\section{"Correspondence:}

wlb_math@163.com

School of Mathematics and

Statistics, Beihua University, JiLin

City, P.R. China

\begin{abstract}
In this paper, we consider a one-dimensional mean curvature equation in Minkowski space and the corresponding one-parameter problem. By using a fixed point theorem of cone expansion and compression of norm type, the existence and multiplicity of positive solutions for the above problems are obtained. Meanwhile, as applications of our results, some examples are given.
\end{abstract}

MSC: $35 \mathrm{~J} 93 ; 34 \mathrm{~B} 16 ; 34 \mathrm{~B} 18$

Keywords: Mean curvature equations; Positive solution; Existence; Multiplicity; Fixed point theorem of cone expansion and compression of norm type

\section{Introduction}

The problem

$$
\operatorname{div}\left(\frac{\nabla v}{\sqrt{1-|\nabla v|^{2}}}\right)=H(x, v) \text { in } \Omega
$$

arises from the study of prescribed mean curvature of a spacelike graph in the flat Minkowski space

$$
L^{N+1}:=\left\{(x, t): x \in \mathbb{R}^{N}, t \in \mathbb{R}\right\}
$$

endowed with the Lorentzian metric $\sum_{i=1}^{N} d x_{i}^{2}-d t^{2}[1]$.

Recently, these kinds of problems have been studied by many authors. In [2], Bereanu et al. considered the Dirichlet problem for the mean curvature equation in Minkowski space of the type

$$
\left\{\begin{array}{l}
\operatorname{div}\left(\frac{\nabla v}{\sqrt{1-|\nabla v|^{2}}}\right)+f(|x|, v)=0 \quad \text { in } B_{R} \\
v=0 \text { on } \partial B_{R}
\end{array}\right.
$$

where $B_{R}=\left\{x \in \mathbb{R}^{N}:|x|<R\right\}$ and $f:[0, R] \times[0, \alpha) \rightarrow \mathbb{R}$ is positive on $(0, R] \times(0, \alpha)$. When $\alpha=R=1, f$ is superlinear at 0 and sublinear at 1 with respect to $\phi(s):=s / \sqrt{1-s^{2}}$, the

(c) The Author(s) 2018. This article is distributed under the terms of the Creative Commons Attribution 4.0 International License (http://creativecommons.org/licenses/by/4.0/), which permits unrestricted use, distribution, and reproduction in any medium, provided you give appropriate credit to the original author(s) and the source, provide a link to the Creative Commons license, and indicate if changes were made. 
authors obtained the existence of classical positive radial solutions of the problem by using the Leray-Schauder degree arguments. In [3], Bereanu et al. studied the positive radial solutions of the one-parameter problem involving the mean curvature operator in Minkowski space of the type

$$
\left\{\begin{array}{l}
\operatorname{div}\left(\frac{\nabla v}{\sqrt{1-|\nabla v|^{2}}}\right)+\lambda\left[\mu(|x|) v^{q}\right]=0 \quad \text { in } B_{R} \\
v=0 \quad \text { on } \partial B_{R}
\end{array}\right.
$$

where $\lambda>0$ is a parameter, $q>1, R>0, \mu:[0,+\infty) \rightarrow R$ is continuous, positive on $(0, \infty)$. Using upper and lower solutions together with Leray-Schauder degree type arguments, the authors proved that there exists $\Lambda>0$ such that the problem has zero, at least one or at least two positive radial solutions according to $\lambda \in(0, \Lambda), \lambda=\Lambda$ or $\lambda>\Lambda$. In [4], Pei and Wang considered the singular Dirichlet problem involving the mean curvature operator in Minkowski space of the form

$$
\left\{\begin{array}{l}
\operatorname{div}\left(\frac{\nabla v}{\sqrt{1-|\nabla v|^{2}}}\right)+f(|x|, v)=0 \quad \text { in } B_{1} \\
v=0 \quad \text { on } \partial B_{1}
\end{array}\right.
$$

and the corresponding one-parameter problem

$$
\left\{\begin{array}{l}
\operatorname{div}\left(\frac{\nabla v}{\sqrt{1-|\nabla v|^{2}}}\right)+\lambda f(|x|, v)=0 \quad \text { in } B_{1} \\
v=0 \quad \text { on } \partial B_{1}
\end{array}\right.
$$

where $f \in C([0,1] \times[0,1),[0,+\infty))$. They provided sufficient conditions for the existence of triple and arbitrarily many positive solutions to the above problems by LeggettWilliams' fixed point theorem. In [5], Pei and Wang considered a strongly singular Dirichlet problem involving the mean curvature operator in Minkowski space of the form

$$
\left\{\begin{array}{l}
\operatorname{div}\left(\frac{\nabla v}{\sqrt{1-|\nabla v|^{2}}}\right)+f(|x|, v)=0 \quad \text { in } B_{1} \\
v=0 \quad \text { on } \partial B_{1}
\end{array}\right.
$$

where $f(r, u)$ is nonnegative and continuous on $(0,1) \times(0,+\infty)$ and may be singular at $r=0$ and/or $r=1$ and strongly singular at $u=0$. The authors presented sufficient conditions which guarantee the existence of positive solutions to the problem by applying the perturbation technique and nonlinear alternative of Leray-Schauder type. In [6], Dai considered the solvability of the Dirichlet problem with mean curvature operator in the Minkowski space

$$
\left\{\begin{array}{l}
\operatorname{div}\left(\frac{\nabla v}{\sqrt{1-|\nabla v|^{2}}}\right)+\lambda f(x, v)=0 \quad \text { in } \Omega \\
v=0 \quad \text { on } \partial \Omega
\end{array}\right.
$$

where $\Omega$ is a general bounded domain of $\mathbb{R}^{N}$. By bifurcation and topological methods, the author determined the interval of parameter $\lambda$ in which the above problem has zero/one/two nontrivial nonnegative solutions according to sublinear/linear/superlinear 
nonlinearity at zero. We refer the reader to [7-15] for the $N$-dimensional mean curvature equation in Minkowski space. In particular, for one-dimensional mean curvature equation with Dirichlet/Neumann/periodic/mixed type boundary conditions in Minkowski space, we refer the reader to [16-24] and the references therein.

Motivated by above work, in this paper, we will consider the one-dimensional mean curvature equations in Minkowski space of the type

$$
\left\{\begin{array}{l}
\left(\frac{u^{\prime}}{\sqrt{1-u^{\prime 2}}}\right)^{\prime}+f(r, u)=0, \quad r \in(0,1) \\
u^{\prime}(0)=0, \quad u(1)=0
\end{array}\right.
$$

and the corresponding one-parameter problem

$$
\left\{\begin{array}{l}
\left(\frac{u^{\prime}}{\sqrt{1-u^{\prime 2}}}\right)^{\prime}+\lambda f(r, u)=0, \quad r \in(0,1) \\
u^{\prime}(0)=0, \quad u(1)=0
\end{array}\right.
$$

where $f \in C([0,1] \times[0,1),[0,+\infty))$. Using a fixed point theorem of cone expansion and compression of norm type, we obtain the existence and multiplicity of positive solutions of the above problems. It is worth to note that, in our work, we do not assume that $f$ is superlinear at 0 and sublinear at 1 with respect to $\phi(s)$. To the best of our knowledge, this is the first paper using a fixed point theorem of cone expansion and compression of norm type to study the above problem.

The rest of the paper is organized as follows. By means of a fixed point theorem of cone expansion and compression of norm type (see [25]), in Sect. 2 we show the existence and multiplicity of positive solutions of (1.1) and (1.2). In Sect. 3, we give some examples to illustrate our results.

\section{Main results}

In order to introduce our main theorem, we need some lemmas.

Simple computations lead to the following lemma.

Lemma 2.1 Let $\phi(s)$ be defined by above. Then $\phi^{-1}(s)=s / \sqrt{1+s^{2}}$ and

$$
\phi^{-1}\left(s_{1}\right) \phi^{-1}\left(s_{2}\right) \leq \phi^{-1}\left(s_{1} s_{2}\right) \leq s_{1} s_{2}, \quad \forall s_{1}, s_{2} \in[0,+\infty)
$$

Lemma 2.2 ([25]) Let E be a Banach space and let $K$ be a cone in E. Assume that $\Omega_{1}$ and $\Omega_{2}$ are bounded open subsets of $E$ such that $0 \in \Omega_{1} \subset \bar{\Omega}_{1} \subset \Omega_{2}$, and let $T: K \cap\left(\bar{\Omega}_{2} \backslash \Omega_{1}\right) \rightarrow K$ be a completely continuous operator such that either

(i) $\|T x\| \leq\|x\|$ for $x \in K \cap \partial \Omega_{1}$ and $\|T x\| \geq\|x\|$ for $x \in K \cap \partial \Omega_{2}$, or

(ii) $\|T x\| \geq\|x\|$ for $x \in K \cap \partial \Omega_{1}$ and $\|T x\| \leq\|x\|$ for $x \in K \cap \partial \Omega_{2}$.

Then $T$ has a fixed point in $K \cap\left(\bar{\Omega}_{2} \backslash \Omega_{1}\right)$.

Take $E=C[0,1]$ with the usual supremum norm $\|\cdot\|$ and the corresponding open ball of center 0 and radius $\rho>0$ will be denoted by $B_{\rho}$. Let

$$
K=\{u \in E: u(r) \text { is concave and nonincreasing on }[0,1], u(1)=0\} \text {. }
$$


Then $K$ is a cone in $E$ and, for all $u \in K$,

$$
u(r) \geq(1-r)\|u\| \quad \text { on }[0,1] .
$$

Now we define a nonlinear operator $T$ on $K \cap B_{1}$ as follows:

$$
(T u)(r)=\int_{r}^{1} \phi^{-1}\left(\int_{0}^{s} f(\tau, u(\tau)) \mathrm{d} \tau\right) \mathrm{d} s, \quad u \in K \cap B_{1}
$$

Clearly, $(T u)(r) \geq 0,(T u)^{\prime \prime}(r) \leq 0$ for all $r \in[0,1],(T u)^{\prime}(0)=(T u)(1)=0$, which implies that $T\left(K \cap B_{1}\right) \subset K$. Moreover, it is easy to show by a standard argument that $T$ is compact on $K \cap \bar{B}_{\rho}$ for all $\rho \in(0,1)$ (see [2]). In addition, it can easily be verified that $u$ is a positive solution of problem (1.1) if $u \in K \cap B_{1}$ is a fixed point of the nonlinear operator $T$.

Now, we state and prove the existence and multiplicity of positive solutions of problem (1.1) and (1.2) by using a fixed point theorem of cone expansion and compression of norm type.

For convenience, we introduce some notations

$$
f_{0}^{J}=\varliminf_{s \rightarrow 0^{+}} \min _{r \in J} \frac{f(r, s)}{\phi(s)}, \quad f^{0}=\varlimsup_{s \rightarrow 0^{+}} \max _{r \in[0,1]} \frac{f(r, s)}{\phi(s)}, \quad f^{1}=\varlimsup_{s \rightarrow 1^{-}} \max _{r \in[0,1]} \frac{f(r, s)}{\phi(s)},
$$

where $J$ be a compact subinterval of $[0,1]$.

Theorem 2.1 Assume that there exists a compact subinterval $:=\left[r_{0}, r_{1}\right] \subset[0,1)$ such that

$$
f_{0}^{J}>\frac{\sigma}{1-r_{1}} \text { and } f^{1}<1
$$

where $\sigma=\frac{1}{\left(r_{1}-r_{0}\right)^{2}}\left(\sqrt{1+\left(r_{1}-r_{0}\right)^{2}}+1\right)$. Then problem $(1.1)$ has at least one positive solution.

Proof Take a number $L$ with $\frac{\sigma}{1-r_{1}}<L<f_{0}^{J}$. Then

$$
\lim _{\delta \rightarrow 0^{+}} L \frac{\phi\left(\left(1-r_{1}\right) \delta\right)}{\phi(\sigma \delta)}=L \frac{1-r_{1}}{\sigma}>1
$$

It follows that there exists $\delta^{*} \in(0,1)$ with $\sigma \delta^{*}<1$ such that

$$
L \frac{\phi\left(\left(1-r_{1}\right) \delta\right)}{\phi(\sigma \delta)}>1, \quad \forall \delta \in\left(0, \delta^{*}\right)
$$

Since $f_{0}^{J}>L$, there exists $\rho \in\left(0, \delta^{*}\right)$ such that

$$
\frac{f(r, s)}{\phi(s)}>L, \quad \forall(r, s) \in J \times(0, \rho] .
$$

Therefore, for each $(r, s) \in J \times\left[\left(1-r_{1}\right) \rho, \rho\right]$ we have

$$
f(r, s)>L \phi(s) \geq L \phi\left(\left(1-r_{1}\right) \rho\right)>\phi(\sigma \rho) .
$$


Now, let $\Omega_{1}=\{u \in E:\|u\|<\rho\}$. Then from (2.1), (2.2) and Lemma 2.1, we obtain

$$
\begin{aligned}
\|T u\| & =\int_{0}^{1} \phi^{-1}\left(\int_{0}^{s} f(\tau, u(\tau)) \mathrm{d} \tau\right) \mathrm{d} s \\
& \geq \int_{r_{0}}^{r_{1}} \phi^{-1}\left(\int_{r_{0}}^{s} f(\tau, u(\tau)) \mathrm{d} \tau\right) \mathrm{d} s \\
& >\int_{r_{0}}^{r_{1}} \phi^{-1}\left(\int_{r_{0}}^{s} \phi(\sigma \rho) \mathrm{d} \tau\right) \mathrm{d} s \\
& \geq \sigma \rho \int_{r_{0}}^{r_{1}} \phi^{-1}\left(s-r_{0}\right) \mathrm{d} s \\
& =\sigma \rho \int_{0}^{r_{1}-r_{0}} \quad \phi^{-1}(s) \mathrm{d} s \\
& =\rho=\|u\|, \quad \forall u \in K \cap \partial \Omega_{1} .
\end{aligned}
$$

Next, we prove that there exists $\varrho \in(\rho, 1)$ such that

$$
\|T u\|<\|u\|, \quad \forall u \in K \cap \partial B_{\varrho} .
$$

In fact, from $f^{1}<1$, there exist $\alpha \in\left(f^{1}, 1\right)$ and $\beta \in(0,1)$ such that

$$
f(r, s) \leq M+\alpha \phi(s), \quad \forall(r, s) \in[0,1] \times[0,1)
$$

where $M=\max \{f(r, s):(r, s) \in[0,1] \times[0, \beta]\}$.

Select $\varrho>0$ with

$$
\max \left\{\rho, \phi^{-1}\left(\frac{M}{1-\alpha}\right)\right\}<\varrho<1
$$

Then from (2.5) we get

$$
\begin{aligned}
\|T u\| & =\int_{0}^{1} \phi^{-1}\left(\int_{0}^{s} f(\tau, u(\tau)) \mathrm{d} \tau\right) \mathrm{d} s \\
& \leq \int_{0}^{1} \phi^{-1}\left(\int_{0}^{s}(M+\alpha \phi(\varrho)) \mathrm{d} \tau\right) \mathrm{d} s \\
& =\int_{0}^{1} \phi^{-1}((M+\alpha \phi(\varrho)) s) \mathrm{d} s \\
& <\phi^{-1}(M+\alpha \phi(\varrho)) \\
& <\phi^{-1}((1-\alpha) \phi(\varrho)+\alpha \phi(\varrho)) \\
& =\varrho=\|u\|, \quad \forall u \in K \cap \partial B_{\varrho},
\end{aligned}
$$

that is, (2.4) holds.

Let $\Omega_{2}=\{u \in E:\|u\|<\varrho\}$. Then from (2.4) we have

$$
\|T u\|<\|u\|, \quad \forall u \in K \cap \partial \Omega_{2} .
$$


Therefore, from (2.3), (2.6) and Lemma 2.2, the operator $T$ has a fixed point $u^{*} \in K \cap$ $\left(\bar{\Omega}_{2} \backslash \Omega_{1}\right)$, which is a positive solution of problem (1.1). This completes the proof of the theorem.

Corollary 2.1 Assume that there exists a compact subinterval $J:=\left[r_{0}, r_{1}\right] \subset(0,1)$ such that

$$
\frac{\sigma}{\left(1-r_{1}\right) f_{0}^{J}}=: \Lambda_{1}<\Lambda_{2}:=\frac{1}{f^{1}}
$$

where $\sigma=\frac{1}{\left(r_{1}-r_{0}\right)^{2}}\left(\sqrt{1+\left(r_{1}-r_{0}\right)^{2}}+1\right)$. Then the one-parameter problem (1.2) has at least one positive solution provided $\Lambda_{1}<\lambda<\Lambda_{2}$.

\section{Theorem 2.2 Assume that}

(i) $f^{0}<1$ and $f^{1}<1$;

(ii) there exist a compact subinterval $\left[r_{0}, r_{1}\right] \subset[0,1)$ and $\rho \in(0,1)$ such that $\sigma \rho \in(0,1)$ and

$$
f(r, s)>\phi(\sigma \rho), \quad \forall(r, s) \in\left[r_{0}, r_{1}\right] \times\left[\left(1-r_{1}\right) \rho, \rho\right],
$$

where $\sigma=\frac{1}{\left(r_{1}-r_{0}\right)^{2}}\left(\sqrt{1+\left(r_{1}-r_{0}\right)^{2}}+1\right)$.

Then problem (1.1) has at least two positive solutions.

Proof Since $f^{0}<1$, there exists $\rho_{0} \in(0, \rho)$ such that

$$
f(r, s)<\phi(s), \quad \forall(r, s) \in[0,1] \times\left[0, \rho_{0}\right]
$$

Let $\Omega_{0}=\left\{u \in E:\|u\|<\rho_{0}\right\}$, then, for $u \in K \cap \partial \Omega_{0}$,

$$
\begin{aligned}
\|T u\| & =\int_{0}^{1} \phi^{-1}\left(\int_{0}^{s} f(\tau, u(\tau)) \mathrm{d} \tau\right) \mathrm{d} s \\
& <\int_{0}^{1} \phi^{-1}\left(\int_{0}^{s} \phi(u(\tau)) \mathrm{d} \tau\right) \mathrm{d} s \\
& \leq \int_{0}^{1} \phi^{-1}\left(\int_{0}^{1} \phi\left(\rho_{0}\right) \mathrm{d} \tau\right) \mathrm{d} s \\
& =\rho_{0}=\|u\| .
\end{aligned}
$$

Let $\Omega_{1}=\{u \in E:\|u\|<\rho\}$. Then from the proof of Theorem 2.1 we obtain

$$
\|T u\|>\|u\|, \quad \forall u \in K \cap \partial \Omega_{1} .
$$

Notice that $f^{1}<1$, it follows from the proof of Theorem 2.1 that there exists $\varrho \in(\rho, 1)$ such that

$$
\|T u\|<\|u\|, \quad \forall u \in K \cap \partial \Omega_{2},
$$

where $\Omega_{2}=\{u \in E:\|u\|<\varrho\}$. Therefore, it follows from (2.7), (2.8), (2.9) and Lemma 2.2 that the operator $T$ has two fixed points $u_{1} \in K \cap\left(\bar{\Omega}_{1} \backslash \Omega_{0}\right)$ and $u_{2} \in K \cap\left(\bar{\Omega}_{2} \backslash \Omega_{1}\right)$, which 
are two distinct positive solutions of problem (1.1). This completes the proof of the theorem.

Remark 2.1 In Theorem 2.2, the condition $f^{0}<1$ in (i) can be replaced by the following condition: there exists $\rho_{0} \in(0, \rho)$ such that

$$
f(r, s)<\phi\left(\rho_{0}\right), \quad \forall(r, s) \in[0,1] \times\left[0, \rho_{0}\right]
$$

The conclusion still holds.

\section{Corollary 2.2 Assume that}

(i) there exist a compact subinterval $\left[r_{0}, r_{1}\right] \subset[0,1)$ and $\rho \in(0,1)$ such that $\sigma \rho \in(0,1)$ and

$$
f(r, s)>0, \quad \forall(r, s) \in\left[r_{0}, r_{1}\right] \times\left[\left(1-r_{1}\right) \rho, \rho\right]=: D,
$$

where $\sigma=\frac{1}{\left(r_{1}-r_{0}\right)^{2}}\left(\sqrt{1+\left(r_{1}-r_{0}\right)^{2}}+1\right)$;

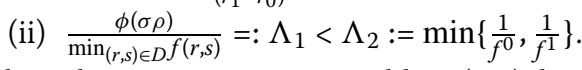

Then the one-parameter problem (1.2) has at least two positive solutions provided $\Lambda_{1}<$ $\lambda<\Lambda_{2}$.

Proof At first, from the assumption (i), we know that $\min _{(r, s) \in D} f(r, s)>0$, and thus $\Lambda_{1}$ is a finite number. We now check that all conditions of Theorem 2.2 are satisfied. From the assumption (ii), for $\lambda<\Lambda_{2}$, we have

$$
\lambda f^{i}<\Lambda_{2} f^{i} \leq 1, \quad i=0,1 .
$$

On the other hand, from assumption (ii), for $\lambda>\Lambda_{1}$, we have

$$
\begin{aligned}
\lambda f(r, s)>\Lambda_{1} f(r, s) & >\frac{\phi(\sigma \rho)}{\min _{(r, s) \in D} f(r, s)} f(r, s) \\
& \geq \frac{\phi(\sigma \rho)}{\min _{(r, s) \in D} f(r, s)} \min _{(r, s) \in D} f(r, s) \\
& =\phi(\sigma \rho), \quad \forall(r, s) \in D .
\end{aligned}
$$

Therefore, by Theorem 2.2, the one-parameter problem (1.2) has at least two positive solutions provided $\Lambda_{1}<\lambda<\Lambda_{2}$.

Theorem 2.3 Assume that there exists a compact subinterval $J:=\left[r_{0}, r_{1}\right] \subset[0,1)$ such that

(i) $f_{0}^{J}=\infty$ and $f^{1}<1$;

(ii) there exists $\rho \in(0,1)$ with $\sigma \rho \in(0,1)$ such that

$$
\begin{aligned}
& \quad f(r, s)>\phi(\sigma \rho), \quad \forall(r, s) \in J \times\left[\left(1-r_{1}\right) \rho, \rho\right], \\
& \text { where } \sigma=\frac{1}{\left(r_{1}-r_{0}\right)^{2}}\left(\sqrt{1+\left(r_{1}-r_{0}\right)^{2}}+1\right) ;
\end{aligned}
$$


(iii) there exists $\rho_{0} \in(0, \rho)$ such that

$$
f(r, s)<\phi\left(\rho_{0}\right), \quad \forall(r, s) \in[0,1] \times\left[0, \rho_{0}\right] .
$$

Then problem (1.1) has at least three positive solutions.

Proof From the assumptions (i), (ii), (iii) and Remark 2.1, the conditions of Theorem 2.2 are all satisfied. Then from the proof of Theorem 2.2, there exist $\rho$ and $\varrho$ with $0<\rho_{0}<\rho<$ $\varrho<1$ such that

$$
\begin{gathered}
\|T u\|<\|u\|, \quad \forall u \in K \cap \partial B_{\rho_{0}}, \\
\|T u\|>\|u\|, \quad \forall u \in K \cap \partial B_{\rho},
\end{gathered}
$$

and

$$
\|T u\|<\|u\|, \quad \forall u \in K \cap \partial B_{\varrho},
$$

where $B_{\rho_{0}}=\left\{u \in E:\|u\|<\rho_{0}\right\}, B_{\rho}=\{u \in E:\|u\|<\rho\}, B_{\varrho}=\{u \in E:\|u\|<\varrho\}$.

Moreover, from $f_{0}^{J}=\infty$ and the proof of Theorem 2.1, there exists $\rho^{*}>0$ with $\rho^{*}<\rho_{0}$, such that

$$
\|T u\|>\|u\|, \quad \forall u \in K \cap \partial B_{\rho^{*}}
$$

where $B_{\rho^{*}}=\left\{u \in E:\|u\|<\rho^{*}\right\}$. Then from Lemma 2.2, problem (1.1) has three distinct positive solutions $u_{0}, u_{1}, u_{2}$ with $u_{0} \in K \cap\left(\bar{B}_{\rho_{0}} \backslash B_{\rho^{*}}\right), u_{1} \in K \cap\left(\bar{B}_{\rho} \backslash B_{\rho_{0}}\right)$ and $u_{2} \in K \cap$ $\left(\bar{B}_{\varrho} \backslash B_{\rho}\right)$.

Corollary 2.3 Assume that there exists a compact subinterval $J:=\left[r_{0}, r_{1}\right] \subset[0,1)$ such that

(i) $f_{0}^{J}=\infty$;

(ii) there exists $\rho \in(0,1)$ with $\sigma \rho \in(0,1)$ such that

$$
f(r, s)>0, \quad \forall(r, s) \in J \times\left[\left(1-r_{1}\right) \rho, \rho\right]=: D_{\rho},
$$

where $\sigma=\frac{1}{\left(r_{1}-r_{0}\right)^{2}}\left(\sqrt{1+\left(r_{1}-r_{0}\right)^{2}}+1\right)$;

(iii) there exists $\rho^{*} \in(0, \rho)$ such that

$$
\begin{aligned}
& \qquad \frac{\phi(\sigma \rho)}{\min _{(r, s) \in D_{\rho}} f(r, s)}=: \Lambda_{1}<\Lambda_{2}:=\min \left\{\frac{1}{f^{1}}, \frac{\phi\left(\rho^{*}\right)}{\max _{(r, s) \in D_{\rho^{*}}} f(r, s)}\right\}, \\
& \text { where } D_{\rho^{*}}:=[0,1] \times\left[0, \rho^{*}\right] .
\end{aligned}
$$

Then the one-parameter problem (1.2) has at least three positive solutions provided $\Lambda_{1}<$ $\lambda<\Lambda_{2}$.

Proof Similar to the proof of Corollary 2.2, it is easy to check that, for $\Lambda_{1}<\lambda<\Lambda_{2}, \lambda f(t, s)$ satisfies all conditions of Theorem 2.3. Then the conclusion hold. 


\section{Examples}

In this section, we give some examples to demonstrate the applications of the our results.

Example 3.1 Consider the following one-dimensional mean curvature equations in Minkowski space of the type

$$
\left\{\begin{array}{l}
\left(\frac{u^{\prime}}{\sqrt{1-u^{\prime 2}}}\right)^{\prime}+\left(r-\frac{1}{2}\right)^{2} \frac{u^{p}}{\left(1-u^{2}\right)^{q}}=0, \quad r \in(0,1), \\
u^{\prime}(0)=0, \quad u(1)=0,
\end{array}\right.
$$

where $0<p<1$ and $q<1 / 2$ are constants.

Let $f(r, s)=\left(r-\frac{1}{2}\right)^{2} s^{p} /\left(1-s^{2}\right)^{q}$ on $[0,1] \times[0,1)$. Take $J=\left[\frac{3}{4}, 1\right]$, it is easy to see that $f_{0}^{J}=\infty$ and $f^{1}=0$. By Theorem 2.1, the problem (3.1) has at least one positive solution. We note that Theorem 1 of [2] cannot guarantee this conclusion since $f\left(\frac{1}{2}, s\right)=0, \forall s \in[0,1)$.

Example 3.2 Consider the following one-parameter problem:

$$
\left\{\begin{array}{l}
\left(\frac{u^{\prime}}{\sqrt{1-u^{\prime 2}}}\right)^{\prime}+\lambda\left|r-\frac{37}{48}\right|^{p} u^{q}=0, \quad r \in(0,1), \\
u^{\prime}(0)=0, \quad u(1)=0,
\end{array}\right.
$$

where $\lambda>0$ be a parameter, $p \geq 0, q>1$ are constants.

Let $f(r, s)=\left|r-\frac{37}{48}\right|^{p} s^{q}$ on $[0,1] \times[0,1)$. It is easy to see that $f^{0}=f^{1}=0$. Choose $\rho=1 / 12$, $r_{0}=1 / 4$ and $r_{1}=3 / 4$. Then $\sigma \rho=(2+\sqrt{5}) / 6 \in(0,1)$,

$$
\phi(\sigma \rho)=\frac{\sigma \rho}{\sqrt{1-(\sigma \rho)^{2}}}=\frac{2+\sqrt{5}}{\sqrt{27-4 \sqrt{5}}}
$$

and

$$
\min _{(r, s) \in D} f(r, s)=\min _{(r, s) \in D}\left|r-\frac{37}{48}\right|^{p} s^{q}=\frac{1}{48^{p+q}},
$$

where $D=\left[\frac{1}{4}, \frac{3}{4}\right] \times\left[\frac{1}{48}, \frac{1}{12}\right]$. Thus from Corollary 2.2, the one-parameter problem (3.2) has at least two positive solutions provided

$$
\lambda>\Lambda_{1}:=48^{p+q} \frac{2+\sqrt{5}}{\sqrt{27-4 \sqrt{5}}} .
$$

Example 3.3 Consider the following one-parameter problem:

$$
\left\{\begin{array}{l}
\left(\frac{u^{\prime}}{\sqrt{1-u^{\prime 2}}}\right)^{\prime}+\lambda g(u)=0, \quad r \in(0,1), \\
u^{\prime}(0)=0, \quad u(1)=0,
\end{array}\right.
$$


where $\lambda>0$ is a parameter and

$$
g(s)= \begin{cases}\frac{1}{10} \sqrt{s}, & s \in\left[0, \frac{1}{96}\right], \\ \frac{2}{5}(360-\sqrt{6})\left(s-\frac{1}{48}\right)+\frac{3}{2}, & s \in\left[\frac{1}{96}, \frac{1}{48}\right], \\ 3456 s^{2}, & s \in\left[\frac{1}{48}, \frac{1}{12}\right], \\ -\frac{276}{5}\left(s-\frac{1}{2}\right)+1, & s \in\left[\frac{1}{12}, \frac{1}{2}\right], \\ \frac{1}{2} \sqrt[4]{\frac{12}{1-s^{2}},} & s \in\left[\frac{1}{2}, 1\right) .\end{cases}
$$

We choose $\rho=1 / 12, r_{0}=1 / 4, r_{1}=3 / 4$ and $\rho^{*}=1 / 96$. It is easy to check that the conditions for Corollary 2.3 are all satisfied, where

$$
\Lambda_{1}=\frac{2(2+\sqrt{5})}{3 \sqrt{27-4 \sqrt{5}}}<1, \quad \Lambda_{2}=\frac{40 \sqrt{6}}{\sqrt{9215}}>1 .
$$

Hence from Corollary 2.3, problem (3.3) has at least three positive solutions when $\lambda \in$ $\left(\Lambda_{1}, \Lambda_{2}\right)$.

\section{Acknowledgements}

The authors thank the referee for valuable suggestions, which led to improvement of the original manuscript.

\section{Funding}

This work was supported by the Education Department of JiLin Province ([2016]45).

\section{Competing interests}

The authors declare that they have no competing interests.

\section{Authors' contributions}

All authors contributed equally to the writing of this paper. All authors read and approved the final manuscript.

\section{Publisher's Note}

Springer Nature remains neutral with regard to jurisdictional claims in published maps and institutional affiliations.

Received: 27 November 2017 Accepted: 21 March 2018 Published online: 27 March 2018

\section{References}

1. Bartnik, R., Simon, L.: Spacelike hypersurfaces with prescribed boundary values and mean curvature. Commun. Math. Phys. 87, 131-152. 1982-83

2. Bereanu, C., Jebelean, P., Torres, P.J.: Positive radial solutions for Dirichlet problems with mean curvature operators in Minkowski space. J. Funct. Anal. 264, 270-287 (2013)

3. Bereanu, C., Jebelean, P., Torres, P.J.: Multiple positive radial solutions for a Dirichlet problem involving the mean curvature operator in Minkowski space. J. Funct. Anal. 265, 644-659 (2013)

4. Pei, M., Wang, L.: Multiplicity of positive radial solutions of a singular mean curvature equations in Minkowski space. Appl. Math. Lett. 60, 50-55 (2016)

5. Pei, M., Wang, L.: Positive radial solutions of a mean curvature equation in Minkowski space with strong singularity. Proc. Am. Math. Soc. 145, 4423-4430 (2017)

6. Dai, G.: Bifurcation and nonnegative solutions for problem with mean curvature operator on general domain. Indiana Univ. Math. J. (in press). http://www.iumj.indiana.edu/IUMJ/Preprints/7546.pdf

7. Bereanu, C., Jebelean, P., Mawhin, J.: Radial solutions for some nonlinear problems involving mean curvature operators in Euclidean and Minkowski spaces. Proc. Am. Math. Soc. 137, 161-169 (2009)

8. Bereanu, C., Jebelean, P., Mawhin, J.: Multiple solutions for Neumann and periodic problems with singular $\phi$-Laplacian. J. Funct. Anal. 261, 3226-3246 (2011)

9. Bereanu, C., Jebelean, P., Mawhin, J.: The Dirichlet problem with mean curvature operator in Minkowski space-a variational approach. Adv. Nonlinear Stud. 14, 315-326 (2014)

10. Coelho, I., Corsato, C., Rivetti, S.: Positive radial solutions of the Dirichlet problem for the Minkowski-curvature equation in a ball. Topol. Methods Nonlinear Anal. 44, 23-39 (2014)

11. Corsato, C., Obersnel, F., Omari, P., Rivetti, S.: Positive solutions of the Dirichlet problem for the prescribed mean curvature equation in Minkowski space. J. Math. Anal. Appl. 405, 227-239 (2013)

12. Dai, G.: Bifurcation and positive solutions for problem with mean curvature operator in Minkowski space. Calc. Var. Partial Differ. Equ. 55, 72 (2016) 
13. Dai, G.: Global bifurcation for problem with mean curvature operator on general domain. Nonlinear Differ. Equ. Appl. 24, 30 (2017)

14. Dai, G., Wang, J.: Nodal solutions to problem with mean curvature operator in Minkowski space. Differ. Integral Equ. 30, 463-480 (2017)

15. Kusahara, T., Usami, H.: A barrier method for quasilinear ordinary differential equations of the curvature type. Czechoslov. Math. J. 50, 185-196 (2000)

16. Bereanu, C., Jebelean, P., Mawhin, J.: Non-homogeneous boundary value problems for ordinary and partial differential equations involving singular $\phi$-Laplacians. Mat. Contemp. 36, 51-65 (2009)

17. Bereanu, C., Jebelean, P., Mawhin, J.: Periodic solutions of pendulum-like perturbations of singular and bounded $\phi$-Laplacians. J. Dyn. Differ. Equ. 22, 463-471 (2010)

18. Bereanu, C., Mawhin, J.: Existence and multiplicity results for some nonlinear problems with singular $\phi$-Laplacian. J. Differ. Equ. 243, 536-557 (2007)

19. Bereanu, C., Mawhin, J.: Multiple periodic solutions of ordinary differential equations with bounded nonlinearities and $\phi$-Laplacian. Nonlinear Differ. Equ. Appl. 15, 159-168 (2008)

20. Bereanu, C., Mawhin, J.: Nonhomogeneous boundary value problems for some nonlinear equations with singular $\phi$-Laplacian. J. Math. Anal. Appl. 352, 218-233 (2009)

21. Brezis, H., Mawhin, J.: Periodic solutions of the forced relativistic pendulum. Differ. Integral Equ. 23, 801-810 (2010)

22. Herlea, D.-R., Precup, R.: Existence, localization and multiplicity of positive solutions to $\phi$-Laplace equations and systems. Taiwan. J. Math. 20, 77-89 (2016)

23. Mawhin, J: Periodic solutions of the forced pendulum: classical vs relativistic. Matematiche 65, 97-107 (2010)

24. Torres, P.: Nondegeneracy of the periodically forced Liénard differential equation with $\phi$-Laplacian. Commun. Contemp. Math. 13, 283-292 (2011)

25. Guo, D., Lakshmikantham, V:: Nonlinear Problems in Abstract Cones. Academic Press, Boston (1988)

\section{Submit your manuscript to a SpringerOpen ${ }^{\circ}$ journal and benefit from:}

- Convenient online submission

- Rigorous peer review

- Open access: articles freely available online

- High visibility within the field

- Retaining the copyright to your article

Submit your next manuscript at $\boldsymbol{\nabla}$ springeropen.com 